\title{
Innovación en las empresas manufactureras de Cuenca
}

\section{Innovation in manufacturing companies in Cuenca}

\author{
Silvia Raquel Mejía Matute ${ }^{1 *}$, Erika Belén García Galarza ${ }^{1}$, Luis Gabriel Pinos Luzuriaga ${ }^{1}$, Luis Bernardo \\ Tonon Ordóñez ${ }^{1}$, Bladimir Proaño Rivera ${ }^{1}$ \\ ${ }^{1}$ Universidad del Azuay, Ecuador \\ *smejia@uazuay.edu.ec
}

DOI: https://doi.org/10.26871/killkanasocial.v5i1.674

\begin{abstract}
Resumen
La mayoría de innovaciones de las empresas se basan en el conocimiento adquirido de fuentes externas como licencias, patentes, actividades de ingeniería industrial, consultorías, laboratorios, universidades entre otros. Este conocimiento de fuentes internas o externas se transforma en capacidades tecnológicas y organizacionales a través de sistemas, rutinas, manuales, liderazgo y gestión. De esta manera, este conocimiento es aprovechado para mejorar los procesos de producción y distribución para disminuir sus costos y mejorar la calidad de los productos. Para ello, las actividades de innovación son importantes ya sea adquiriendo conocimiento externo o generando el propio, pues la innovación no es un proceso lineal y permite retroalimentar, corregir, adaptar y mejorar los procesos y productos. En tal sentido, el objetivo del presente estudio es conocer las principales estrategias de innovación de procesos y las actividades de innovación que aplican las empresas manufactureras de Cuenca. Para ello, se utilizó una metodología cuantitativa a través de un cuestionario que se aplicó en 122 empresas seleccionadas a través de un muestreo probabilístico del total de empresas cuencanas dentro del sector manufacturero registradas en la Superintendencia de Compañías, Valores y Seguros en el año 2019. Como resultado se obtuvo que las empresas grandes desarrollan tanto actividades como procesos de innovación sólidos en cambio las pequeñas y medianas empresas tienen mayores dificultades para desarrollar capacidades de innovación particularmente para disminuir sus costos. Ciertamente, los avances innovativos disruptivos son un reto para las empresas y el sistema productivo de los países. Los cambios tecnológicos influyen trascendentalmente en el crecimiento económico y consecuentemente en el desarrollo tanto de capital físico como capital humano. Por ello, las organizaciones para mantenerse en el mercado deben alcanzar un mínimo requerido de avances innovativos y así ser más competitivas.
\end{abstract}

Palabras clave: actividades de innovación, indicadores de innovación, innovación de procesos, manufacturas, método de la diferencia.

\begin{abstract}
Most of the innovations of companies are based on the knowledge acquired from external sources such as licenses, patents, industrial engineering activities, consultancies, laboratories, universities, among others. This knowledge from internal or external sources is transformed into technological and organizational capabilities through systems, routines, manuals, leadership and management. In this way, this knowledge is used to improve production and distribution processes to reduce costs and improve the quality of products. For this, innovation activities are important either by acquiring external knowledge or generating their own, since innovation is not a linear process and allows feedback, correction, adaptation and improvement of processes and products. In this sense, the objective of this study is to know the main process innovation strategies and the innovation activities applied by manufacturing companies in Cuenca. For this, a quantitative methodology was used through a questionnaire that was applied in 122 companies selected through a probabilistic sampling of the total of Cuenca companies within the manufacturing sector registered in the Superintendency of Companies, Securities and Insurance in 2019. As a result, it was obtained that large companies develop activities such as solid innovation processes, while small and medium-sized companies have greater difficulties in developing innovation capacities, particularly to reduce their costs. Certainly, disruptive innovative advances are a challenge for companies and the productive system of countries. Technological changes have a transcendental influence on economic growth and consequently on the development of both physical capital and human capital. Therefore, organizations to stay in the market must achieve a minimum required of innovative advances and thus be more competitive.
\end{abstract}

Keywords: difference method, innovation activities, innovation indicators, process innovation, manufacturing. 


\section{Introducción}

La innovación es un elemento trascendental para las organizaciones empresariales en todos los sectores económicos, pues alientan la sostenibilidad, evolución y crecimiento económico. Porter (1990) plantea que, mantenerse a la vanguardia de los distintos estándares mundiales para la innovación es de gran relevancia para la competitividad de una nación, pues conjuntamente con el capital humano forjan las ventajas competitivas de las economías industriales.

Según Lederman et al. (2014) manifiestan que las empresas latinoamericanas crecen de manera desacelerada, por la falta de innovación, lo que hace evidente que para que las empresas crezcan deberían innovar de forma continua. Su estudio mostró puntuaciones bajas en temas de innovación para los países latinoamericanos, debido principalmente a que no se introducen nuevos productos con gran frecuencia, no se invierte en mejores prácticas para la gestión de la organización, la investigación y el desarrollo es baja, y la actividad de patentes está por debajo de cifras referenciales.

La innovación permite aumentar la productividad y competitividad de un país. En el ranking del Índice Global de Innovación en Latinoamérica los países que más resaltan para el año 2019, son Chile en el puesto 51, Costa Rica 54 y México 56 que han realizado valiosos aportes para sus avances en innovación. Ecuador también se ha encaminado en la senda de la innovación a través del esfuerzo del estado y los actores sociales para invertir en capital humano, investigación, y tecnología. Igualmente, las empresas han destinado inversiones en capacitaciones al personal, gastos de investigación y desarrollo e importaciones de tecnologías. Sin embargo, Ecuador se ubica en el puesto 99 de Innovación (World Intellectual Property Organization, 2019).

En el año 2019 la economía ecuatoriana cerró con el Producto Interno Bruto (PIB) de 71.879 millones de dólares en términos constantes, es decir creció en una cuantía porcentual del 0,1 . Por su parte, el VAB del sector manufacturero se incrementó en 1,5\% con respecto al año 2018. También se puede acotar que en el sector de la manufactura, los cantones que más aportaron al VAB industrial en el año 2018 son Quito 27,99\%, Guayaquil 27,87 \%, Durán 6,54 \% y Cuenca 5,65 \% (Banco Central del Ecuador, 2020).

Según Robalino (2020), Cuenca tiene grandes proyecciones para los próximos 20 a 30 años en lo que concierne a las industrias de: línea blanca, cerámica, muebles de madera, neumáticos, plástico, cartón y papel entre otras. Por ello, se considera que existe cierta adaptabilidad a las nuevas necesidades, cultura de consumo y a las nuevas tecnologías que se van desarrollando en la ciudad.

Es así que cabe preguntarse si, ¿en qué aspectos las actividades y procesos de innovación de las empresas industriales están mejorando y contribuyendo al progreso de sus capacidades de innovación? La viabilidad del estudio se centra en que la ciudad de Cuenca, se ubica como el cuarto cantón en aporte al Valor Agregado Bruto de las manufacturas en el país y su historia está ligada a la actividad artesanal. Así mismo, el Instituto Nacional de Estadística, INEC (2018), registró para este año que la industria de manufacturas generó 303.674 fuentes de empleo, con una remuneración promedio de USD. 809,04 mensuales en este sector. Es importante dinamizar el sector manufacturero, a través de la innovación en sus procesos y actividades para que de esa manera su competitividad y productividad lleguen a niveles en donde la producción y ventas se incrementen, y así pueda continuar siendo una fuente importante de generación de empleo en el país.

El objetivo de este estudio es conocer las principales estrategias de innovación de procesos y las actividades de innovación que aplican las empresas de manufacturas de Cuenca - Ecuador. Para lo cual se consideró tanto los procesos como las actividades de innovación. La metodología utilizada fue cuantitativa a través de una encuesta aplicada en las empresas que están registradas en este sector como activas en la Superintendencia de Compañías, Seguros y Valores, para lo cual se utilizó el método de muestreo aleatorio estratificado. El cuestionario contiene preguntas sobre el marco innovativo de las empresas. Para el análisis de resultados, se calcularon indicadores que permiten evaluar las empresas mejor y peor posicionadas en este campo.

El artículo se organiza de la siguiente manera: seguido a la introducción, en la sección uno se detalla los antecedentes de trabajos empíricos realizados sobre innovación empresarial. Posteriormente, en la sección dos se describen los componentes de la metodología utilizada; en la sección tres se dan a conocer los resultados obtenidos de la aplicación del cuestionario, y los indicadores de innovación en el proceso y de actividades de innovación por medio del método de la diferencia; y por último, en la sección cuatro se presentan las conclusiones del estudio.

\section{Antecedentes}

La Organización para la Cooperación y el Desarrollo Económicos, OECD, y Eurostat (2005) en el Manual de Oslo, conciben a la innovación como la introducción en el mercado, de un bien o proceso nuevo o significativamente mejorado y agregan lo referido al desarrollo de nuevas técnicas de organización y comercialización. La acción misma de innovar tiene que ver con la aplicación de distintos tipos de conocimientos, competencias, capacidades y recursos, para alcanzar una ventaja competitiva por medio de la eficiencia en disminución de costos o por el desarrollo de nuevos productos o cambios significativos en los existentes (Fagerberg, 2013).

Según el OECD (2015) en el Manual de Frascati, la innovación es la transformación de una idea en un producto nuevo o mejorado, que se introduce en el mercado o en procesos de producción nuevos o mejorados que son utilizados dentro del sector económico o industrial, o en el comercio.

Schumpeter (1912), inicia el análisis de la innovación con la Teoría del Desenvolvimiento Económico, la cual 
concluye que el emprendedor es el motor del proceso de las transformaciones continuas en una empresa de producción que determina el avance no lineal de la sociedad. El desenvolvimiento económico se da por medio de fenómenos que generan desequilibrios en el entorno industrial, empresarial y comercial, el factor principal de este desenvolvimeinto surge por parte de los empresarios que son propiamente agentes de innovaciones, por lo que las necesidades de los consumidores han quedado en un segundo plano para definir el rumbo del desenvolvimiento económico.

Las innovaciones que se generan y desarrollan por los empresarios protagonizan el desarrollo económico planteado por Schumpeter; así mismo al momento de la implementación de innovaciones beneficiarán a algunos sectores y perjudicarán a otros, este suceso se denomina "Destrucción Creativa" (Schumpeter, 1947).

Seguido a las teorías propuestas por Schumpeter, surgen las teorías de Nelson \& Winter (1982), quienes divisaron de una forma distinta la teoría planteada, aludiendo que los avances e innovaciones en las empresas son resultados de un proceso de adaptación al entorno económico y social, con la visión de permanencia en los mercados.

Para Pavitt (1984), a través de una clasificación sectorial por tipo de industria, se encontró que para cada empresa, la estrategia de innovación es distitna, pues esta dependerá de las características del sector en el que se encuentre, siendo éstas carcaterísticas determinantes para la intensidad de innovación de las empresas de la propia industria. Así también, afirma que para los sectores con altos niveles de producción intensiva, se esperaría que exista una alta proporción de recursos que se encaminen a procesar en mayor proporción innovaciones.

Posteriormente, Aghion y Howitt (1992), siguiendo el pensamiento schumpeteriano proponen el modelo de "Crecimiento a través de la Destrucción Creadora" por medio del cual se genera el proceso de innovación, según el enfoque endógeno. El modelo propone innovaciones verticales, las cuales se van reemplazando y tornando obsoletas a las innovaciones anteriores. Se plantea también, que cada innovación origina un monopolio en la economía, sobre la producción del insumo de las eventuales invenciones, por lo que las empresas o industrias que llevan a cabo las innovaciones obtienen beneficios extraordinarios, hasta que otra empresa o industria cree una nueva innovación y la desplace. A tales beneficios extraordinarios se les puede denominar ventaja competitiva, como lo establece Porter (1990).

En cambio Lall (1992), no basa su propuesta en innovaciones verticales o disruptivas, sino que tiene por objetivo implantar transformaciones en empresas que siguen los procesos de cambios de las emprendedoras, tratando de imitar las innovaciones realizadas por éstas, ya que de esa manera se evitará quedar excluidas del mercado.

Para Fudenberg \& Tirole (1995), resalta la relevancia del posicionamiento con respecto a la competencia, pues, las empresas de un determinado sector realizan in- novaciones para defender su posición con respecto a sus competidores, así también pueden obtener nuevas ventajas competitivas. Las empresas reaccionan innovando para no perder cuota de mercado, y obtener una posición estratégica en el mercado con relación a sus competidoras.

Nelson y Winter (1982); Lundvall (2007); realizaron estudios de la influencia de las organizaciones externas sobre las actividades innovadoras de las empresas, dando como principales hallazgos la transferencia y difusión de las ideas, experiencias, conocimientos, y otros elementos. La información circula mediante canales y redes que conectan los marcos social, político y cultural que conducen y delimitan las actividades y la capacidad de innovación. Es así que, la innovación es denominada como un proceso dinámico que acumula aprendizaje e interacciones.

Para Kline y Rosenberg (2009), recalcan la incertidumbre en los progresos futuros en cuanto a la innovación, conocimientos, tecnología, mercados y demanda de productos, ya que son difíciles de prever. La adopción de nuevos o mejorados productos y procesos, la utilización de nuevos métodos de comercialización y organización, son sucesos repletos de incertidumbre.

Entonces, la innovación de procesos se concibe como la relacionada con la forma o los métodos de elaboración de los distintos procesos de producción o de distribución que realiza la organización. Esto implica cambios significativos en técnicas, materiales y programas informáticos (OECD y Eurostat, 2005). Las innovaciones de procesos tienen como objetivo disminuir los costos unitarios de producción o distribución, incrementar los niveles de calidad, y sobretodo producir productos nuevos o mejorados significativamente.

Para Rodríguez (1999); Malaver y Vargas (2004); la innovación se asocia con la tecnología, ya que para la tecnología es indispensable iniciar con un proyecto innovador. En el mismo contexto, Drucker (2000), concibe la innovación como la actividad dirigida a la generación y aplicación de nuevos conocimientos. Sin embargo, no solo implica tecnología sino también "Disciplina de la innovación", que significa buscar oportunidades internas: acontecimientos inesperados, incongruencias, cambios de procesos y de mercados; y, oportunidades externas: cambios demográficos y de percepción, así como también búsqueda de nuevos conocimientos y experiencias.

En el presente artículo se muestran indicadores de innovación, los cuales según Lugones (2008), son herramientas importantes para la toma de decisiones en las empresas, pues de esa manera se definen las estrategias competitivas. A la vez las empresas mediante los indicadores de innovación pueden revisar y reformular las estrategias y sus prácticas en el ámbito tecnológico, organizacional y comercial. De esta manera, Kalthoff (2001) plantea que, la innovación es una variable estratégica para obtener ventaja competitiva.

Para Hernández y Sánchez (2003), el cambio tecnológico y las actividades de innovación no están homo- 
géneamente distribuidos entre los más diversos sectores industriales de la economía.

Las actividades de innovación se refieren a la creación de nuevos conocimientos como adquirir, adaptar o desarrollar conocimientos existentes, así como también a las distintas maneras de incrementar las capacidades productivas y tecnológicas de la organización ya sea por medio de la implementación de nuevos equipamentos, de recursos humanos innovados, entre otros. Dentro de este aspecto se incluyen las siguientes variables: Investigación y Desarrollo (I+D), por medio de métodos internos de la empresa o ya sean por contratos a agentes externos; adquisición de tecnología incorporada y desincorporada en la empresa, las cuales pueden ser en equipos, hardware, software, licencias, patentes, entre otras; contrato de consultor y asistencia técnica; actividades de ingeniería y diseño industrial; capacitación del personal; actividades de marketing.

Cabe recalcar la importancia de la medición de los procesos y actividades innovativas, pues mediante la evidencia empírica, estas están directamente relacionadas con mejoras genuinas de competitividad, crecimiento económico y mejoras en los niveles de bienestar de la sociedad.

Kline y Rosenberg (1986), realizan investigaciones con la concepción de la innovación como proceso, en las cuales se deduce que esta no es una sucesión lineal de hechos, con una estructura y orden predeterminado, sino que se trata de un sistema de retroalimentación, corrección e interacción entre los conocimientos, habilidades y funciones de los trabajadores, cuya experiencia y habilidades se refuerzan mutuamente con todo el equipo de trabajo y tal actividad se acumula. Para potenciar estos sucesos se debe propiciar una articulación a redes de innovación. El procedimiento mencionado se origina como soporte y propulsor del conocimiento generado, especialmente en los departamentos de I+D, o surge como una necesidad de la sociedad.

Por otro lado, en el caso colombiano, según Malaver y Vargas (2004), utilizan a la innovación como resultado, lo que se traduce en el producto de los procesos innovados que se hayan trabajado, por lo cual es de vital importancia establecer indicadores que esclarezcan la situación y descripción de los resultados obtenidos de la implementación de procesos innovadores. En el estudio, los indicadores se clasificaron según su tipo, es decir en innovaciones en los productos, en los procesos, organizacionales y en el ámbito comercial, según su grado, en radical o incremental, y según su alcance en los mercados, los cuales pueden ser local, regional, nacional e internacional.

En otros estudios aplicados, tales como el de Chamónica y Gómez (2017), se realiza un análisis de la industria aeroespacial para México, Canadá y Estados Unidos en la aplicación de innovación y desarrollo, e inversión extranjera directa en el periodo 2005 - 2015. Para ello, se ejecuta un modelo econométrico cuyo resultado fue que las variables explicativas de investigación y desarrollo e inversión extranjera directa, influyen positivamente en el desarrollo de la tecnología en la industria.

En el caso ecuatoriano, Astudillo y Briozzo (2016), destacan un estudio comparativo entre las micro, pequeñas y medianas empresas manufactureras de Ecuador y Argentina relacionadas con la innovación. Los resultados fue que las empresas manufactureras argentinas y ecuatorianas son diferentes en torno al progreso de la innovación de los productos y procesos. Aunque, las actividades de innovación como el gasto de investigación y desarrollo sobre ventas, registro de patentes y apoyo del sector público, destacan porque se desarrollan simultáneamente en ambos países.

\section{Metodología}

La construcción del procedimiento se enfocó en el análisis global de los elementos de innovación tanto en actividades como en procesos, de manera que se posibilite a los empresarios del sector llevar a cabo una valoración organizacional y procedimental en cuanto a la innovación generada en las empresas cuencanas del sector manufacturero. Para ello, se utilizó una metodología cuantitativa basada en una encuesta aplicada en las empresas que se registran en situación legal activas en la Superintendencia de Compañías, Valores y Seguros (2019) dentro de la actividad de manufacturas en la ciudad de Cuenca. Este universo de estudio era de 328 empresas.

El tamaño de la muestra se calculó utilizando el $90 \%$ de nivel de confianza y un margen de error del $6 \%$ para poblaciones finitas. El resultado fue una muestra con 122 empresas. Se utilizó el muestreo aleatorio estratificado para distribuir la muestra, porque se consideró que la población tiene un comportamiento diferente en las subpoblaciones; por ello, se trabajó con estratos y no dominios, pues esto permite aumentar la eficiencia de las estimaciones y contribuye a la administración eficiente de grandes encuestas (Valliant, 2000, como se citó en Gutiérrez, 2016). Entonces, se tomó en cuenta en la distribución, por un lado, al tamaño de las empresas (grandes, medianas, pequeñas y microempresas); y por otro lado, el subsector según la Clasificación Industrial Internacional Uniforme (CIIU 4.0) nivel 2. Para Cuenca se tienen los siguientes subsectores de las manufacturas: C10, C11, C13, C14, C15, C16, C17, C18, C19, C20; C21, C22, C24.

Cabe señalar que inicialmente se determinó una muestra con un nivel de confianza del $95 \%$ y un margen de error del $5 \%$, sin embargo se tuvo limitaciones para obtener respuestas por parte de las empresas, entre otras razones porque ya habían contestado otras encuestas, o se dieron cambios de domicilio y registros de contacto. La encuesta fue realizada en el último trimestre de 2019, en principio se envió el cuestionario por correo electrónico y se acordaron fechas para que los encuestadores visitemos los establecimientos y se entrevistó a la persona delegada, gerentes o propietarios.

El diseño del cuestionario indagó tanto en los procesos de innovación como en las actividades de innovación. 
Tabla 1: Indicadores utilizados para la recolección de información

\begin{tabular}{l} 
Tema de las preguntas \\
\hline Innovación en el proceso: (*) \\
1) Métodos de manufactura o producción \\
2) Logística, métodos de entrega o distribución de insumos \\
3) Sistemas de mantenimiento u operaciones de compra, contabilidad, computación \\
4) Se realizó actividades de innovación, pero fueron interrumpidas \\
5) Si pero las actividades de innovación están aún en curso \\
Actividades de adaptación y mejora: \\
1) I+D externa \\
2) I+D interna \\
3) Adquisición de maquinaria, equipo y software destinado a la producción de \\
productos o procesos nuevos o mejorados de manera significativa para la innovación. \\
4) Adquisición de conocimientos externos para innovación (Patentes, Derechos de \\
Propiedad Intelectual, Licencias, Know How) \\
5) Capacitación para la innovación (formación interna o externa de personal destinado \\
específicamente al desarrollo o introducción de productos o procesos nuevos o \\
mejorados de manera significativa) \\
6) Diseño (forma y aspecto de producto)
\end{tabular}

Fuente: La construcción de la tabla es propia a partir de la Encuesta de Estado de las Empresas de Cuenca, 2020.

(*) El valor de las respuestas fue 1 . Si tiene y 0 . No tiene.

La información fue procesada en el paquete estadístico SPSS. Primero, se elaboraron tablas, gráficos y estadísticos descriptivos. Luego, se construyó el índice de innovación de procesos y de actividades para lo cual, se otorgó el valor de uno a las respuestas afirmativas y de cero a las negativas. Luego, se procedió a obtener un cociente entre las opciones elegidas sobre las opciones posibles de toda la industria. Finalmente, se comparó este promedio de la industria con los índices obtenidos ya sea por tamaño o por subsector de la manufactura. De esa manera, se constituyeron los índices. Estos se graficaron en un plano cartesiano, donde se puede observar que, si el índice está cercano a uno o ciento por ciento (indicador 5), entonces son mejores las capacidades innovativas de la empresa y si está cercano a cero (indicador 0-1), en cambio ocurre lo contrario.

En el caso de las empresas manufactureras cuencanas, se trató de diagnosticar el nivel de innovación, ya que para ingresar al sector deben tener un mínimo requerido que forzosamente demandan algún tipo de innovación para posicionarse y poder funcionar en la industria.

La estrategia de análisis es una descripción de lo ocurrido en el sector manufactuero de Cuenca en relación a los aspectos que permiten dar cuenta de los procesos de innovación y las actividades de innovación y mediante la estadistica descriptiva se construyó un índice para cada uno. Adicionalmente, se utilizó el método de la diferencia, que consiste en la selección de casos positivos y negativos del objeto de estudio lo cual visibiliza la variación existente en la variable dependiente. La lógica de inferencia de este procedimiento es que todos los casos que presentan el resultado de interés deben también compartir una característica explicativa común, mientras que los casos negativos deben carecer de este atributo (Mill, 1973).

Los casos positivos son empresas manufactureras que poseen innovaciones tanto en procesos como actividades innovadoras sólidas, y los casos negativos son las empresas de la industria manufacturera con capacidades débiles de innovación en procesos y actividades.

Al seleccionar los casos positivos y negativos dentro del sector, se infiere las características y las diferencias entre las empresas con mejores capacidades innovativas. Esta información podrá ser de gran utilidad para la industria para que se evalúen y puedan realizar seguimiento de su nivel de inserción en el ámbito de la innovación. De esta manera, se puede establecer si las capacidades son determinantes en la posición actual de las empresas, para que existan posibilidades de escalamiento y mejora para los casos negativos o con capacidades de innovación débiles, para que puedan imitar o seguir a las más fuertes y llegar a un mejor posicionamiento en la innovación del sctor y de la economía del país. En el largo plazo se esperaría estar incursionando en la innovación disruptiva, pero se puede empezar con acciones inmediatas como adaptaciones y mejoras que respondan a los cambios en el mercado.

\section{Resultados}

Descripción de los procesos y actividades de innovación de las empresas estudiadas

La innovación de procesos y las actividades son factores claves para incrementar la productividad; razón por la cual, las empresas invierten tanto en capital humano como en maquinaria, equipos y tecnología en general. De esta manera, se preguntó a los empresarios en una escala del 1 al 5 , siendo 1 poco importante y 5 muy importante, como resultado se obtuvo que, 52,5\% de las empresas en Cuenca consideran importante la inversión en trabajadores cualificados para el crecimiento del negocio, el 10,7\% considera moderadamente importante y el $36,8 \%$ opina que la inversión en trabajadores cualificados es muy importante. En cambio, para el caso de la inversión en propiedades, planta o equipos el 50,8\% consideró muy importante, el $36,9 \%$ importante y el $11,5 \%$ moderadamente importante, se tuvo un $0,8 \%$ de encuestas sin esta respuesta.

Los equipos tecnológicos de producción que utilizan en el proceso de las empresas investigadas el 70,5\% usan equipos de accionamiento manual, 4,1\% equipos automáticos, y $0,8 \%$ digitales y $24,6 \%$ no contesta esta pregunta.

Otra de las características de mayor alcance en la innovación es la introducción de nuevos o mejorados bienes o servicios al mercado, se encontró para este caso de estudio que en los últimos tres años el 37,7\% de empresas manufactureras han incluido significativamente y no lo hizo el $45,9 \%$ y $16,4 \%$ no contesta. También, se conoció que aproximadamente $25,4 \%$ de las empresas desarrollaron innovaciones de bienes por sí solas; es decir, no se basaron en el apoyo o aporte de otras empresas de su grupo, proveedores, otras instituciones, universidades u otras entidades.

El 13,9\% de las empresas manufactureras manifestaron que los productos nuevos o mejorados significativamente representaron el $50 \%$ de las ventas totales de su negocio.

En la tabla 2, se destaca que las empresas han realizado innovación en los procesos, principalmente en sistemas 
de mantenimiento u operación de compra, contabilidad y computación con un $72,1 \%$, así también el $61,5 \%$ de las empresas encuestadas han innovado sus procesos en métodos de manufactura o producción.

Tabla 2: Implicación de desarrollo de innovación en los procesos

\begin{tabular}{lcc}
\hline Innovación en los Procesos (*) & $\begin{array}{c}\text { No } \\
\text { Porcentaje }\end{array}$ & $\begin{array}{c}\text { Si } \\
\text { Porcentaje }\end{array}$ \\
\hline Métodos de Manufactura o Producción & 36,1 & 61,5 \\
Logística, métodos de entrega o distribución de insumos. & 52,5 & 44,3 \\
$\begin{array}{l}\text { Sistemas de Mantenimiento u Operaciones de Compra, } \\
\text { Contabilidad, Computación. }\end{array}$ & 24,6 & 72,1 \\
Actividades de Innovación, pero han sido Interrumpidas & 50,0 & 47,5 \\
Actividades de Innovación que aún se encuentran en Curso & 36,1 & 61,5 \\
\hline
\end{tabular}

Fuente: La construcción de la tabla es propia a partir de la Encuesta de Estado de las Empresas de Cuenca, 2020.

(*) La suma entre las respuestas No y Si es inferior a 100 porque el valor que falta son los casos de empresas que no ofrecieron esta respuesta.

En lo que concierne a las actividades de innovación desarrolladas, se destaca que el $72,9 \%$ de las empresas han realizado innovaciones en el diseño de sus productos, así mismo el $71,3 \%$ han logrado realizar investigación y desarrollo externo. En la figura 1, se puede observar con detalle las actividades de innovación que realizan las empresas, también se observa que las actividades de I+D internas son las que menos se realizan.

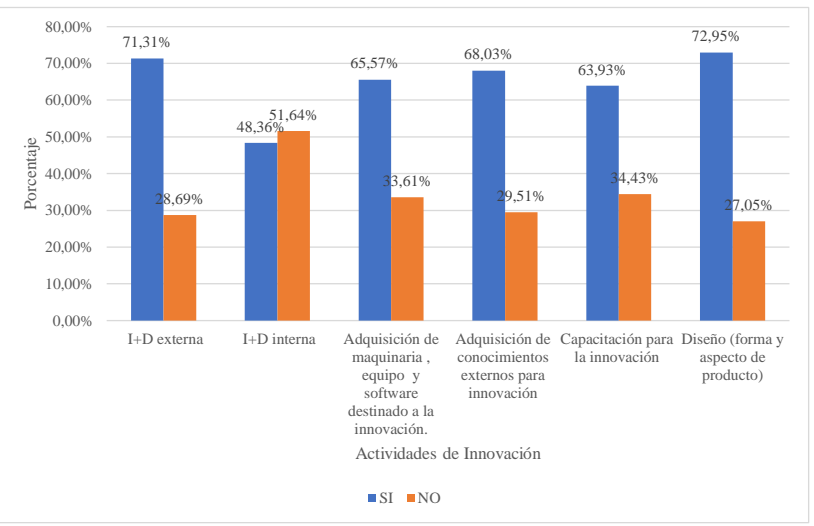

Figura 1: Desarrollo de actividades de innovación. Elaboración propia a partir de datos de la encuesta Estado de las Empresas en Cuenca, 2020

En la tabla 3, se puede observar que según este estudio el $59,0 \%$ de las empresas utilizaron métodos formales de propiedad intelectual como la marca para proteger sus innovaciones en bienes. El 37,7\% utilizó patentes para proteger las innovaciones en servicios.
Tabla 3: Porcentaje de empresas que utilizaron la marca y patentes como métodos formales de propiedad intelectual para proteger sus innovaciones

\begin{tabular}{lcc}
\hline & $\begin{array}{c}\text { La Marca } \\
\text { (Porcentaje) }\end{array}$ & $\begin{array}{c}\text { Patentes } \\
\text { (Porcentaje) }\end{array}$ \\
\hline Si uso en bienes & 59.0 & 26.2 \\
Si uso en servicios & 10.7 & 37.7 \\
Si uso en bienes y & 27.9 & 27.0 \\
servicios & 1.6 & 4.1 \\
Si uso en procesos & 0.8 & 4.1 \\
No uso & $\mathbf{1 0 0 . 0}$ & $\mathbf{1 0 0 . 0}$ \\
\hline Total &
\end{tabular}

Fuente: La construcción de la tabla es propia a partir de la

Encuesta de Estado de las Empresas de Cuenca, 2020

El 87,7\% de las empresas introdujeron nuevas prácticas en la organización de procedimientos, el 59,0\% adaptaron nuevos métodos para la organización de responsabilidades laborales y toma decisiones, y el 79,5\% realizaron nuevos métodos en la organización de relaciones externas.

\subsection{Innovaciones en Procesos}

Como resultado de la complejidad de identificar, desarrollar e implementar innovaciones en los procesos para incrementar la productividad, las empresas cada vez más recurren a fuentes externas de conocimiento. En la Figura 2 , se encuentra en el eje vertical el número de empresas visitadas para este estudio y en el eje horizontal el indicador de innovación de procesos, el cual denota 5 niveles, siendo 1 el indicador que denota las capacidades más débiles de innovaciones en proceso, y por lo contrario 5 son las empresas que tienen las más sólidas capacidades de innovación en los procesos. De esta manera, se puede evidenciar que, las empresas ubicadas en el lado izquierdo son consideradas con capacidades débiles de innovación y en el lado derecho están las empresas calificadas con fuertes y sólidas capacidades de innovación, según el método de la diferencia.

Se tiene que, las empresas del sector manufacturero de Cuenca se ubican en mayor medida en el cuadrante superior del plano cartesiano, es decir, si bien es cierto no poseen capacidades sólidas de innovación en los procesos, si cuentan con grandes avances innovativos dentro de sus procesos, tales como mejores métodos de producción, nuevos y mejorados esquemas de manejo de logística en métodos de entrega y distribución de insumos y la opción de sistemas de mantenimiento y operaciones de compra es la más utilizada. Por ejemplo, las empresas 119, 63, 50, 44, 17, 9, 2; y, 37 presentan capacidades solidas de innovación en los procesos; en tanto que, las empresas 85 y 74 tienen capacidades débiles en este aspecto. 


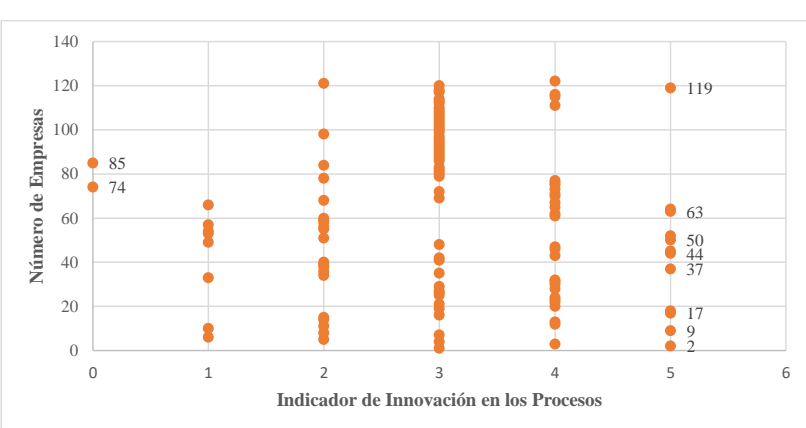

Figura 2: Índice de Innovación de Procesos de las Empresas de Manufacturas de Cuenca. Construcción propia a partir de la Encuesta Estado de las Empresas en Cuenca, 2020

Las empresas con capacidades más sólidas de innovación en los procesos no tienden a ser de un solo tamaño, sino que son de todo tamaño. También, se encontró que estas capacidades existen en la diversidad de subsectores de la manufactura. Por ejemplo, se puede mencionar a las empresas más destacadas que pertenecen a la fabricación de otros productos minerales no metálicos, fabricación de muebles, fabricación de metales comunes, elaboración de productos alimenticios, y fabricación de productos de informática, electrónica y óptica.

Por otro lado, las empresas con capacidades débiles de innovación en los procesos son pequeñas y microempresas pertenecientes a los subsectores económicos de fabricación de productos farmacéuticos, sustancias químicas medicinales y productos botánicos de uso farmacéutico, y fabricación de productos de caucho y plástico. Ninguna empresa grande presenta capacidades débiles de innovación en procesos.

Se puede recalcar que las tres empresas con mayor solidez en la innovación de procesos se destacan por tener muchos años de experiencia en el mercado, tienen personal administrativo con postgrados y obreros con estudios tecnológicos. Adicionalmente, en los últimos tres años han introducido bienes nuevos o mejorados significativamente y han utilizado métodos formales de propiedad intelectual para proteger sus invenciones. En cambio, las empresas con capacidades débiles de innovación se caracterizan por tener menor experiencia en el mercado, dificultades para disminuir los costos de producción, los desperdicios y las fallas de fabricación.

\subsection{Actividades de innovación}

Respecto a las actividades de innovación, siguiendo los mismos criterios de lectura del gráfico anterior, se puede observar en la Figura 3 se puede observar las empresas ubicadas en el lado izquierdo son consideradas con capacidades débiles de innovación en las actividades que desarrollan; y, en el lado derecho están las empresas consideradas con sólidas capacidades.

De manera general, se demuestra un panorama bastante alentador pues solamente el $3 \%$ de las empresas manufactureras cuencanas están por debajo de la mitad del plano cartesiano, y la gran mayoría de las empresas se encuentran en el cuadrante superior, lo que cabe destacar que un gran número de estas cumplen con los estándares de actividades innovadoras dentro de su organización para mejoras en investigación y desarrollo sobre todo externas.

En el caso del sector manufacturero, solamente una empresa se encuentra diferenciada en el lado izquierdo, se trata de la empresa 66 que cuenta con una deficiente capacidad de actividades innovadoras.

En cambio, se destacan las empresas 117, 64, 55, 53, 44, 10; y, 4 con capacidades sólidas en actividades de innovación. Se puede resaltar entonces que las empresas con fortalezas en sus actividades de innovación es una característica de las empresas de cualquier tamaño, ya que se tienen desde microempresas hasta empresas grandes del sector. Así mismo, ningún subsector de la manufactura ya sea alimentos, textiles, minerales, químicos y otros han consolidado sus actividades de innovación. Por ejemplo, en el mismo sector de alimentos se encuentran unas empresas con fortalezas y otras con debilidades. Sin embargo, en los sectores donde predominan las empresas con actividades de innovación fuertes son: la fabricación de otros productos minerales no metálicos, la fabricación de muebles, la reparación e instalación de maquinaria y equipo.

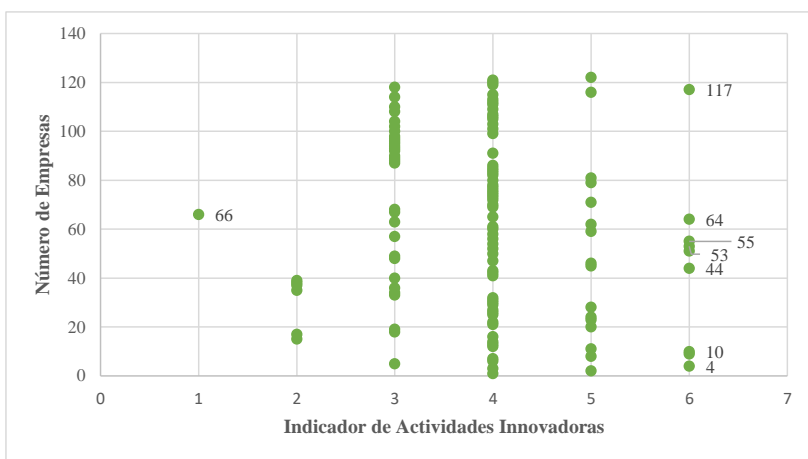

Figura 3: Índice de Actividades Innovadoras de las empresas de Manufacturas de Cuenca. Construcción propia a partir de la encuesta Estado de las Empresas en Cuenca, 2020.

\section{Conclusiones}

El aporte fundamental de este estudio radica en generar información de base que permite cubrir los vacios de datos sobre un aspecto trascendente en el desarrollo productivo del Ecuador, particularmente de Cuenca en lo referido a la Innovación Empresarial.

Se destaca que las empresas manufactureras de Cuenca en su mayoría valoran como muy importante a la inversión en capital físico $(50,8 \%)$ y en menor proporción $(36,8 \%)$ a la inversión en trabajadores cualificados. Sin embargo, la tecnología de producción que se utiliza es 70,5\% basada en equipos de accionamiento manual, $4,1 \%$ equipos 
automáticos y tan sólo $0,8 \%$ en las nuevas tecnologías digitales.

Otro aspecto que se resalta es que en los últimos tres años, la innovación en procesos que han implementado las empresas de este estudio son; en primer lugar, en sistemas de mantenimiento, operaciones de compra, contabilidad y de computación (72,1\%); en segundo lugar, los métodos de manufactura o producción $(61,5 \%)$; y en tercer lugar, la logística y los métodos de entrega y distribución (44,3\%). Lo que permite concluir que se ha puesto mayor énfasis en innovaciones organizacionales y relacionadas con el sistema administrativo que en el propio proceso productivo.

En lo concerniente a las actividades de innovación las que resaltan son relacionadas con el diseño del producto $(72,9 \%)$, la investigación y desarrollo externo a la empresa $(71,3 \%)$ y la adquisición de conocimientos externos $(68,0 \%)$. Así mismo, la mayoría está utilizando métodos formales de propiedad intelectual a través del registro de la marca y patentes.

Finalmente, se puede evidenciar en este estudio que todas las grandes empresas manufactureras de Cuenca muestran capacidades de innovación de procesos fuertes aunque también existen pocas microempresas que también siguen estas estrategias y tienen estas fortalezas. Del mismo modo, las actividades de innovación fuertes son una característica de las empresas grandes, especialmente las relacionadas con la identificación, adquisición e implementación de innovaciones externas. También, el estudio permite concluir que ninguna rama de la manufactura de Cuenca tiene actividades e innovaciones de procesos consolidadas, aunque se puede evidenciar que existen industrias líderes dentro de los sectores de fabricación de otros minerales no metálicos, la fabricación de muebles, y la reparación e instalación de maquinarias y equipos.

Ciertamente, estos resultados ponen de manifiesto que las capacidades de innovación de procesos y actividades de innovación de las empresas de manufacturas de Cuenca son vulnerables y dependientes de las innovaciones tecnológicas externas al tejido empresarial y los propios procesos de ciencia y tecnología del país. También, se puede evidenciar que los procesos manuales son los que predominan y por ello es necesario conocer mejor las capacidades tecnológicas de las empresas, aún más en un momento en que la tecnología avanza a una revolución digital y es importante el trabajo en red. En tal virtud, es necesario aunar esfuerzos para la conformación de sistemas de innovación y tecnología donde los actores sociales como el estado y las universidades tengan un rol más activo e inmerso con la industria.

Por ello, se espera que este trabajo sirva de herramienta de consulta para la toma de decisiones de empresarios y actores sociales y como referente para futuras investigaciones sobre innovación en las empresas.

\section{Referencias Bibliográficas}

Fagerberg, J. (2013). Innovation a New Guide (n. ${ }^{\circ}$
20131119).

World Intellectual Property Organization. (2019). The Global Innovation IndeX 2019: Creating Healthy Lives - The Futures of Medical Innovation.

Recibido: 29 de enero de 2021

Aceptado: 15 de marzo de 2021 